\title{
Differential Inductive Sensing System using a Differential Transformer with Shielded and Split Primary Coil
}

\author{
Marc Berger, Anne Zygmanowski and Stefan Zimmermann \\ Institute of Electrical Engineering and Measurement Technology, Leibniz University Hannover, Appelstr. 9A, \\ 30167 Hannover, Germany \\ Contact: berger@geml.uni-hannover.de
}

\section{Introduction}

In this work, we show a differential transformer for the determination of the electrical and dielectric properties of a sample using a split primary coil and magnetic shielding through a highly permeable ferrite layer for improved sensitivity. Preliminary results show an increase in sensitivity of $12.2 \%$ compared to a transformer with one primary coil and without shielding.

Differential transformers are widely used in sensing technologies. Typically, a differential transformer consist of three coils located on a ferrite core. The center coil is the primary coil and is excited by an ac voltage or an ac current. Due to the excitation, a primary magnetic flux is generated. The outer coils are the secondary coils and are connected differentially in series. In many applications, the ferrite core is movable. This allows a very precise displacement, force or pressure sensor to be realized [1-8].

Another field of application results with a ferrite core fixed symmetrically to the coils. If a sample is placed closer to one of the two secondary coils, its electrical and dielectric properties can be measured. Fig. 1 a) shows such a differential transformer schematically. The primary magnetic flux induces eddy and displacement currents into the sample depending on its permittivity and conductivity. These currents cause a secondary magnetic flux. Due to the lower distance to one of the secondary coils compared to the other, a higher voltage is induced into the closer coil. Thus, an output voltage $U_{0}$ is generated that can be measured at the terminals of the differentially connected secondary coils. [9] shows the possibility to separate $U_{0}$ into a real part depending on the permittivity of the sample and into an imaginary part depending on the conductivity of the sample. Because of the differential connection and the symmetrical setup, the primary magnetic flux induces a voltage in the upper and the lower secondary coil with the same magnitude but different sign, resulting in an output voltage of the connected secondary coils of zero. This allows the weak eddy and displacement currents to be measured with a high resolution. Such a differential transformer with fixed ferrite core can be used for determining the biomass in a bioreactor, the tissue properties or for continuous monitoring of blood properties - e.g. the sodium concentration during the dialysis treatment [914]. [15] has even shown the possibility of measuring directly through a tubing, making it very promising for applications such as continuous and contactless monitoring of sodium in the blood during dialysis treatment. The sodium concentration measurement in blood is realized via blood conductivity measurement, since sodium has the strongest impact on the plasma conductivity (therefore the measuring frequency must be below the $\beta$-dispersion, which is at about $1 \mathrm{MHz}$ ) [16-23].
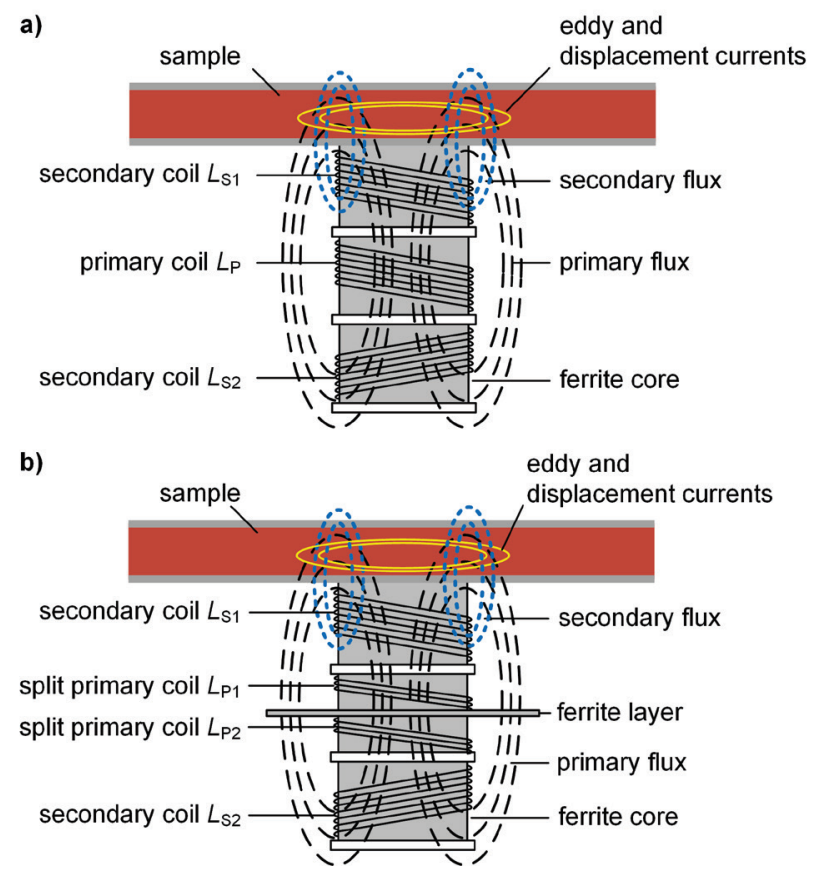

Fig. 1: (a) Schematic depiction of a differential transformer in single-primary coil configuration consisting of the secondary coils $L_{\mathrm{S} 1}$ and $L_{\mathrm{S} 2}$ connected differentially in series and the primary coil $L_{\mathrm{P}}$. (b) Differential transformer with two split primary coils $L_{\mathrm{P} 1}$ and $L_{\mathrm{P} 2}$. A ferrite layer is located between the two primary coils to shield the secondary flux from $L_{\mathrm{S} 2}$.

In order to achieve high sensitivity, it is an advantage to place the primary coil as close as possible to the sample, as this causes the sample to be penetrated strongly by the primary magnetic flux, and thus high eddy and displacement currents are induced in the sample. This increases the field strength of the secondary flux. However, due to the symmetrical design of the transformer, the secondary coil $L$ s2 must also be placed closer to the sample. As a result, this secondary coil is also penetrated by more secondary 
flux. Due to the differential connection of the two secondary coils, this has a negative effect on the sensitivity. In [12] it was therefore shown how to set the optimum distance between the coils.

To further attenuate the secondary magnetic flux at the position of the lower secondary coil, this paper presents a new approach with a primary coil split into two coils and a ferrite layer added between the two primary coils so that the secondary field is shielded from the secondary coil $L_{s}$. A schematic illustration of this setup can be seen in Fig. 1 b). This setup improves the sensitivity of the differential transformer by 12.2 \% compared to the setup shown in Fig. 1 a).

\section{Methods and Materials}

\section{Concept}

The differential transformer with split and shielded primary coils is compared to a PCB (printed circuit board) differential transformer from [12] using one single primary coil $L_{\mathrm{p}}$. This single primary coil transformer has $n_{S}=542$ turns for each secondary coil and $n_{\mathrm{P}}=42$ turns for the primary coil. The distance between the upper and lower secondary coils is $17.5 \mathrm{~mm}$. The primary coil is exited with a voltage $U_{P}=1 \mathrm{VPP}$ (peak to peak) at a frequency of $155 \mathrm{kHz}$. Since the sensitivity of the differential transformer depends on the ratio of the secondary inductance $L_{s}$ to the primary inductance $L_{P}$, and only the effect of the shielding is to be investigated here, this ratio should not be changed by splitting the single primary coil $L_{\mathrm{P}}$ into two primary coils $L_{\mathrm{P} 1}$ and $L_{\mathrm{P} 2}$. This condition can be achieved by two basic configurations.

First, each of the two primary coils can be the same as in the single primary differential transformer. That means both the upper primary coil $L_{\mathrm{P} 1}$ and the lower primary coil $L_{\mathrm{P} 2}$ have 42 turns each. Each coil is excited with $U_{\mathrm{P}}=1 \mathrm{VPP}$ at $155 \mathrm{kHz}$. This corresponds to a parallel configuration of both coils. The mutual inductance $M$ between the primary coils is

$$
M=k \cdot \sqrt{L_{P 1} L_{P 2}},
$$

where $k$ is the coupling factor $(0 \leq k \leq 1)$ [24]. The total inductance $L_{\text {para }}$ of the coupled primary coils with the same winding direction that are connected in parallel is given by [24] as

$$
L_{\text {para }}=\frac{L_{P 1} L_{P 2}-M^{2}}{L_{P 1, \text { para }}+L_{P 2, \text { para }}-2 M} .
$$

If equation (1) is substituted into equation (2) and assuming that $L_{P 1}$ and $L_{P 2}$ are very well coupled via the ferrite core $(k \rightarrow 1)$ and $L_{P 1}=L_{P 2}=L_{P}$, the total inductance $L_{\text {para }}$ is given as

$$
L_{\text {para }}=\lim _{k \rightarrow 1} \frac{L_{P}^{2}-k^{2} L_{P}^{2}}{2 L_{P}-2 k L_{P}}=L_{P}
$$

This gives the same inductance for the two primary coils connected in parallel and coupled via the ferrite core as for the single primary coil differential transformer.
Second, each primary coil now has a reduced number of turns to $n_{P}=21$. Both coils $L_{P 1}$ und $L_{P 2}$ are now connected in series. The total excitation voltage is $U_{P}=1 \mathrm{VPP}$ at $155 \mathrm{kHz}$. Thus, compared to the single primary coil transformer the total number of turns of the primary coils is unchanged. Since the inductance of a coil depends quadratic on the number of turns, the inductances of the individual coils is $L_{p 1}=L_{p 2}=L_{p} / 4$. For magnetically coupled coils connected in series with the same winding direction, the total inductance $L_{\text {seri }}$ results according to [24] to

$$
L_{\text {seri }}=L_{P 1}+L_{P 2}+2 M=\frac{1}{4} L_{P}+\frac{1}{4} L_{P}+\frac{1}{2} k \cdot L_{P} .
$$

Again, assuming that $k$ tends to one, it follows from equation (4) that $L_{\text {seri }}=L_{p}$.

Thus, it is expected that both configurations - the series connection with halved number of turns per primary coil and the parallel connection of two coils, which are identical to the single primary coil differential transformer - provide the same results. Both configurations are compared in the following using numerical simulations.

\section{Simulations}

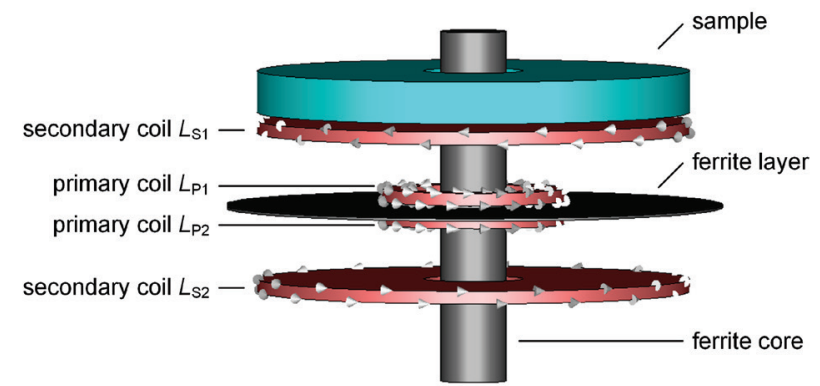

Fig. 2: CST-EM Studio simulation model for simulation of the differential transformer with split and shielded primary coil. The white cones indicate the winding direction.

The ferrite layer has a thickness of $0.3 \mathrm{~mm}$, a radius of $30 \mathrm{~mm}$ and a relative permeability of $\mu_{\mathrm{r}}=300$.

The increase in sensitivity by inserting a ferrite layer with a high relative permeability between the split primary coil is simulated for the two configurations described above using CST-EM Studio. The model corresponds to the PCB differential transformer with two primary coils $L_{P 1}$ and $L_{P 2}$ and is shown in Fig. 2 . The number $n_{s}$ of the turns of each secondary coil is 542 . The distance between the upper and the lower secondary coil is also the same as with the single primary coil transformer $(17.5 \mathrm{~mm})$. To simulate the parallel connection of $L_{P 1}$ and $L_{P 2}$, each primary coil has 42 turns and is wound in the same direction. Both primary coils are excited with a voltage of $1 \mathrm{VPP}_{\mathrm{PP}}$ at $155 \mathrm{kHz}$. To simulate the series configuration of both coils, the number of turns of $L_{P_{1}}$ and $L_{P 2}$ is reduced to 21 each, while exciting each coil with $0.5 V_{P P}$ at $155 \mathrm{kHz}$. Between the two primary coils $L_{\mathrm{P} 1}$ and $L_{\mathrm{P} 2}$ a $0.3 \mathrm{~mm}$ thick ferrite layer with a radius of $30 \mathrm{~mm}$ and a relative permeability $\mu_{r}=300$ is located. The relative 
permeability of the ferrite core is $\mu_{r}=300$ as well. In order to investigate the exact impact of the ferrite layer, each simulation is performed with and without the ferrite layer between $L_{\mathrm{P} 1}$ and $L_{\mathrm{P} 2}$.

As mentioned before, the imaginary part of the output voltage $U_{0}$ depends on the conductivity of the sample. To determine the sensitivity, the conductivity of the sample is varied between $1 \mathrm{~S} / \mathrm{m}$ and $2 \mathrm{~S} / \mathrm{m}$.

\section{Experimental Setup}

The PCB differential transformer with split and shielded primary coil is compared to the PCB differential transformer with the single primary coil from [12]. For both transformers, the secondary coils have 542 turns and are placed with a distance of $17.5 \mathrm{~mm}$ to each other. The excitation voltage is in each case $1 \mathrm{VPP}$ at $155 \mathrm{kHz}$.

As already shown by the theoretical preliminary considerations, and later also proven by the simulation results, the configurations of series connection with halved number of turns $n_{\mathrm{P}} / 2$ or the parallel connection with $n_{P}$ turns are equivalent. Hence, the experimental investigations are only realized in the parallel configuration. A photograph of the differential transformer with split and shielded primary coil is shown in Fig. 3.

The primary coils $L_{\mathrm{P} 1}$ and $L_{\mathrm{P} 2}$ have 42 turns each and are connected in parallel. In the single primary coil configuration, which is used as a reference, $L_{p}$ also has 42 turns.

DI-water (deionized-water) solutions with different sodium chloride $(\mathrm{NaCl})$ concentrations are used as a sample with varying conductivity. The concentration range is increased from $100 \mathrm{mmol} / \mathrm{L}$ in $10 \mathrm{mmol} / \mathrm{L}$ steps up to $150 \mathrm{mmol} / \mathrm{L}$. The sensitivity is given by the slope of the linear regression of the imaginary part of the output voltage $U_{\text {o. }}$.

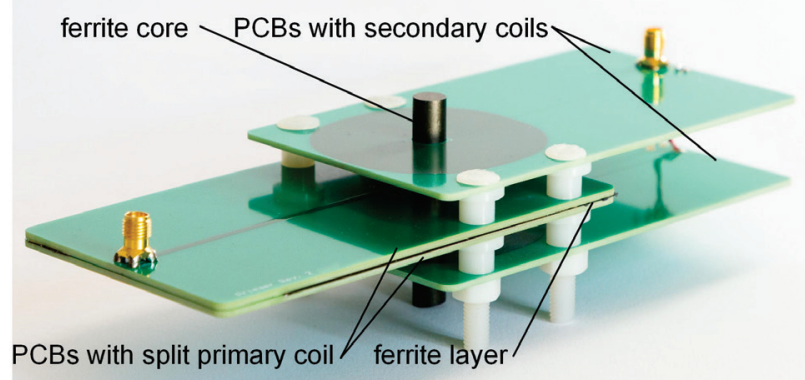

Fig. 3: Photograph of a differential transformer with split and shielded primary coil used for the experimental investigations. Each coil is realized on a separate printed circuit board (PCB). Here, the primary coils $L_{\mathrm{P} 1}$ and $L_{\mathrm{P} 2}$ have the same number of turns $n_{\mathrm{P}}=42$ as $L_{\mathrm{P}}$ of the single primary coil differential transformer from [12]. $L_{\mathrm{P} 1}$ and $L_{\mathrm{P} 2}$ are connected in parallel.

\section{Results}

\section{Simulations}

Initially, the simulations were conducted with two primary coils in series and in parallel configuration without ferrite layer. The results of this simulation are shown in Fig. 4 (parallel configuration: blue line; series configuration: red dashed line).

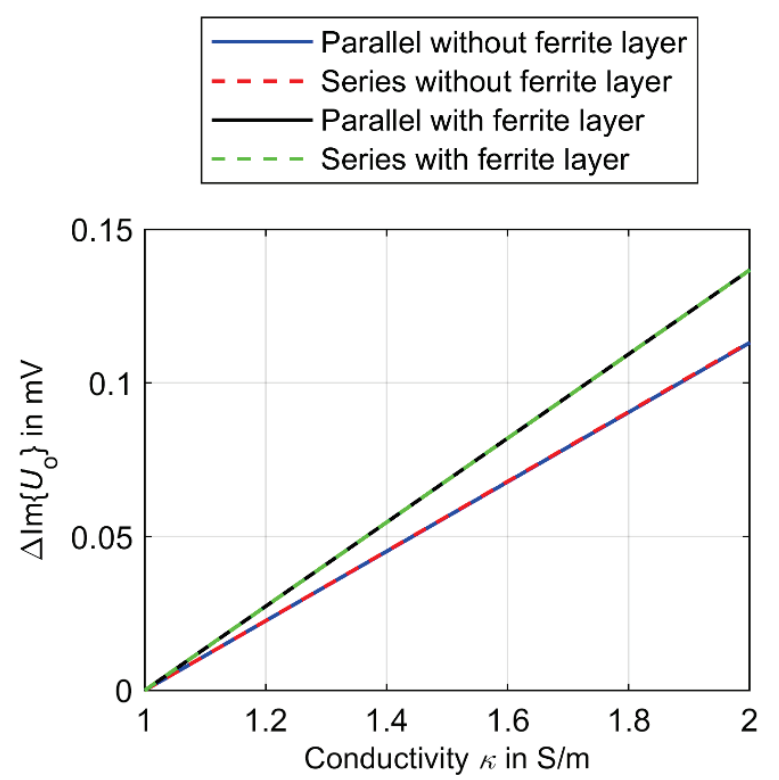

Fig. 4: Simulated variation of the imaginary part of the output voltage $U_{\mathrm{o}}$ depending on the conductivity of the sample using the simulation model from Fig. 2. For the parallel configuration, $n_{\mathrm{P}}$ is 42 turns and the excitation of each coil is $1 \mathrm{~V}_{\mathrm{PP}}$. For the series configuration $n_{\mathrm{P}}=21$ turns and the excitation is $0.5 \mathrm{~V}_{\mathrm{PP}}$ per primary coil. Both configurations were simulated with and without a $0.3 \mathrm{~mm}$ thick ferrite layer between the primary coils. The relative permittivity of the ferrite layer is $\mu_{\mathrm{r}}=300$.

As expected from the theoretical considerations, both configurations have almost the same sensitivity. For the parallel configuration, the simulated sensitivity is $113.2 \mu \mathrm{V} / \mathrm{S} / \mathrm{m}$ and for the series connection, the simulated sensitivity is $113.4 \mu \mathrm{V} / \mathrm{S} / \mathrm{m}$.

Afterwards, the $0.3 \mathrm{~mm}$ thick ferrite layer with a radius of $30 \mathrm{~mm}$ and a $\mu_{\mathrm{r}}=300$ was added between $L_{\mathrm{P} 1}$ and $L_{\mathrm{P} 2}$. The simulated sensitivity is now increased for both configurations to $136.8 \mu \mathrm{V} / \mathrm{S} / \mathrm{m}$ (series configuration: green dashed line; parallel configuration: black line). Thus, the sensitivity increases by about $20 \%$ adding the ferrite layer.

\section{Measurements}

As can be seen in Fig. 5, the differential transformer with split and shielded primary coil has a sensitivity of $215.7 \mathrm{mV} / \mathrm{mol} / \mathrm{L}$ (red), while the differential transformer in single coil configuration has lower sensitivity of $192.2 \mathrm{mV} / \mathrm{mol} / \mathrm{L}$ (blue). Thus, in the experiment, the sensitivity of the differential transformer with split 
and shielded primary coils is $12.2 \%$ higher compared to the single primary coil differential transformer.
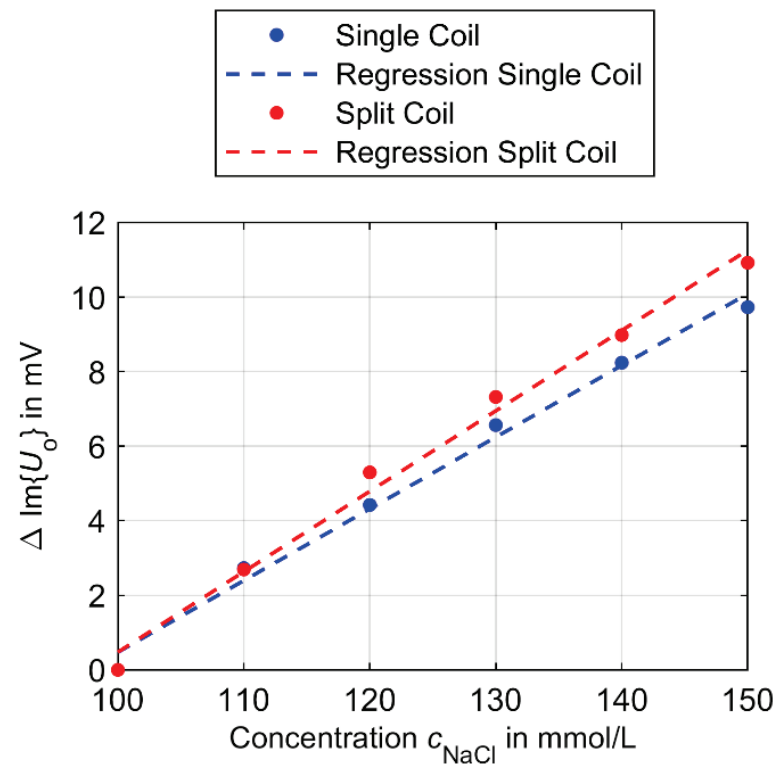

Fig. 5: Comparison between the differential transformer with a single primary coil from [12] (blue) and the differential transformer with a split and shielded primary coil from Fig. 3 (red). The dots are the measured variation of the imaginary part of the output voltage $U_{\mathrm{o}}$ depending on the $\mathrm{NaCl}$ concentration of the sample in DI-water. The dashed lines are the linear regressions to the measured values (the slopes represent the sensitivities).

\section{Conclusion}

In this paper, a concept for sensitivity enhancement of a differential transformer is presented. To achieve this, the primary coil of the transformer is split into two coils and a ferrite layer with a high relative permittivity is added between the two primary coils. Thus, the lower secondary coil being further away from the sample than the upper secondary coil is shielded more from the secondary flux. Due to the differential configuration of the secondary coils, this has a positive effect on the sensitivity.

There are two basic configurations for the connection of the split primary coils, allowing the total inductance of the primary coil not to be changed compared to a single primary coil differential transformer, so that an increase in sensitivity is solely attributed to the shielding. On the one hand, the same coil as for a single primary coil differential transformer can be used twice. These coils must then be connected in parallel. On the other hand, it is also possible to halve the number of turns of the coils and connect both coils in series.

Experimental investigations have shown that the differential transformer with split and shielded primary coils has a sensitivity of $215.7 \mathrm{mV} / \mathrm{mol} / \mathrm{L}$, exceeding the sensitivity of a single primary coil differential transformer by $12.2 \%$.

\section{References}

[1] A. Danisi, A. Masi, R. Losito, Performance Analysis of the Ironless Inductive Position Sensor in the Large Hadron Collider Collimators Environment, Sensors (Basel, Switzerland) 15 (2015) 28592-28602.

[2] C. Loughlin, Sensors for Industrial Inspection, Springer Netherlands, Dordrecht, 1993.

[3] M.J. Usher, Sensors and Transducers, Macmillan Education UK, London, 1985.

[4] W. Petchmaneelumka, W. Koodtalang, V. Riewruja, Simple Technique for Linear-Range Extension of Linear Variable Differential Transformer, IEEE Sensors J. (2019) 1.

[5] J. Fraden, Handbook of Modern Sensors, Springer New York, New York, NY, 2010.

[6] K.V. Santhosh, B.K. Roy, A Smart Displacement Measuring Technique Using Linear Variable Displacement Transducer, Procedia Technology 4 (2012) 854-861.

[7] A.D. Woolfson, D.F. McCafferty, S.P. Gorman, P.A. McCarron, J.H. Price, Design of an apparatus incorporating a linear variable differential transformer for the measurement of type III bioadhesion to cervical tissue, International Journal of Pharmaceutics 84 (1992) 6976.

[8] W.H. Aellig, A new technique for recording compliance of human hand veins, British journal of clinical pharmacology 11 (1981) 237243.

[9] T. Reinecke, P. Biechele, M. Sobocinski, H. Suhr, K. Bakes, D. Solle, H. Jantunen, T. Scheper, S. Zimmermann, Continuous noninvasive monitoring of cell growth in disposable bioreactors, Sensors and Actuators B: Chemical 251 (2017) 1009-1017.

[10] M. Berger, D. Niebuhr, M. Allers, S. Zimmermann, Continuous Non-Invasive Sodium Monitoring in Extracorporeal Circuits: 17th International Meeting on Chemical - IMCS (2018) 683-684.

[11] M. Berger, F. Sellering, H. Rohrich, H. Mansour, T. Perl, S. Zimmermann, A Differential Transformer for Noninvasive Continuous Sodium Monitoring During Dialysis Treatment: 2019 IEEE SENSORS (2019) 1-4.

[12] M. Berger, A. Zygmanowski, F. Sellering, H. Röhrich, T. Perl, H. Mansour, S. Zimmermann, Contactless and continuous sodium concentration monitoring during continuous renal replacement therapy, Sensors and Actuators B: Chemical 320 (2020) 128372.

[13] J Netz, E Forner, S Haagemann, Contactless impedance measurement by magnetic induction - a possible method for investigation of brain impedance, Physiological Measurement 14 (1993) 463-471. 
[14] P. P. Tarjan, R. McFee, Electrodeless Measurements of the Effective Resistivity of the Human Torso and Head by Magnetic Induction, IEEE Transactions on Biomedical Engineering BME-15 (1968) 266-278.

[15] M. Berger, A. Zygmanowski, S. Zimmermann, Differential Inductive Sensing System for Truly Contactless Measuring of Liquids' Electromagnetic Properties in Tubing, Sensors 21 (2021) 5535.

[16] H. Beving, L.E.G. Eriksson, C.L. Davey, D.B. Kell, Dielectric properties of human blood and erythrocytes at radio frequencies $(0.2-10$ $\mathrm{MHz}$ ): dependence on cell volume fraction and medium composition, Eur Biophys $\mathrm{J} 23$ (1994) 207-215.

[17] M. Stubbe, J. Gimsa, Maxwell's mixing equation revisited: characteristic impedance equations for ellipsoidal cells, Biophysical journal 109 (2015) 194-208.

[18] H.P. Schwan, Electrical properties of tissue and cell suspensions, Advances in biological and medical physics 5 (1957) 147-209.

[19] H.P. Schwan, Electrical properties of blood and its constitutents: Alternating current spectroscopy, Blut 46 (1983) 185-197.

[20] F. Montiel, P. Aimar, P. Montoriol, Continuous monitoring of sodium concentration in blood during haemodialysis by a selective membrane and conductivity sensor, Sensors and Actuators B: Chemical 27 (1995) 465-467.

[21] F. Locatelli, S. Di Filippo, C. Manzoni, Relevance of the conductivity kinetic model in the control of sodium pool, Kidney international 58 (2000) S89-S95.

[22] A. Tura, S. Sbrignadello, E. Mambelli, P. Ravazzani, A. Santoro, G. Pacini, Sodium concentration measurement during hemodialysis through ion-exchange resin and conductivity measure approach: In vitro experiments, PloS one 8 (2013) e69227.

[23] F. Locatelli, V. La Milia, L. Violo, L. Del Vecchio, S. Di Filippo, Optimizing haemodialysate composition, Clinical kidney journal 8 (2015) 580-589.

[24] M. Vömel, D. Zastrow, Aufgabensammlung Elektrotechnik 2, Vieweg+Teubner Verlag, Wiesbaden, 1998. 